\title{
$O$-Glycosylation in the Solid to Solid State
}

\author{
Myeongwon Seo, ${ }^{*, a}$ Chongwhan Won. Yongrae Hong, ${ }^{b}$ Soonjae Chang. Bosup Hahn, and Fumio Toda ${ }^{\dagger}$ \\ Department of Chemistry, Ajou University, Stwon 442-749, Korea. "E-mail: mwseo(alldong.com \\ ${ }^{r}$ Department of Chemistry, Factily of Science, Okayama University of Science, Okayama 700-0005, Japan \\ Received Febriary 15, 2007
}

Key Words : Glycosylation, Solid to solid state, Anhydro sugars

Glycosylation in the solid to solid state produced glycosides in a simple, mild and stereoselective fashion, and this methodology could serve as an addition to existing glycosylation procedures.

A large number of efficient glycosylation procedures have been developed; most of these protocols are being carried out in solution. ${ }^{1}$ However, there is still a continuing demand for an appreciable process in terms of mildness, efficacy and stereocontrol.

In our earlier work, ${ }^{2}$ we demonstrated that $N$-glycosylation in the solid to solid state provided glycopyranosyl-uracil or -thymine with an enhancement in selectivity, and that this method could serve as a viable alternate to existing solutions and/or fusion procedures. Extending of this methodology, we now report that $O$-glycosylation in the solid to solid state produced glycoside with excellent stereoselectivity. The glycosylation was simply produced by grinding the glycosyl donor and acceptor in the presence of the promoter with a mortar and pestle for $30 \mathrm{~min}$ under argon atmosphere in a glove box. When necessary, the reaction mixture was further ground in a ball mill for $24 \mathrm{hr}$. The results of $O$-glycosylation obtained from the solution, and present methods for the comparison between the two are summarized in the Table 1.

Tetra- $O$-acetyl- $\alpha$-D-glucopyranosyl bromide 1a, 1,2anhydro-3,4,6-tri- $O$-benzyl- $\alpha$-D-glucopyranose $\mathbf{1 b}^{3}$ and $1,2-$ antydro-3,4,6-tri- $O$-benzyl- $\beta$-D-mannopyranose $1 c^{4}$ used as glycosyl donors, and methanol 2a, 1,2;3,4-di- $O$-isopropylidene- $\alpha$-D-galactopyranose $2 \mathrm{~b}$ and methyl 2,3-di-Omethyl- $\alpha$-D-glucopyranoside 2c used as the glycosyl acceptor are shown in the following Scheme I.

Because of its high hygroscopic nature and the difficulty of handling of 1a due to moisture present in the air, we also tested $1 \mathrm{~b}$ and $1 \mathrm{c}$ as the glycosyl donor instead of $1 \mathrm{a}$ in this study. We found these 1,2-anhydrosugars gave better results in the formation of a disaccharide with greatly reduced reaction times and enhanced yjelds of product. Thus, the reaction of $1 b$ with $2 c$ in the solid to solid state for $30 \mathrm{~min}$ produced $43 \%$ of glucoside, while the same reaction in solution for $20 \mathrm{hr}$ produced only $35 \%$ of the product.

In the presence of Amberlite IRC-50, a cationic form of jonic exchange resin, and in the absence of promoter, $1 \mathrm{~b}$ also reacted with $2 \mathrm{c}$ and formed the corresponding disaccharide,

${ }^{2}$ Present address: Research Laborotories, Ildong Pharmaceutical Co., Lid., Yongin 449-910, Korea

bPresent address: Crystal Genomics, Inc., Seoul 138-736, Korea

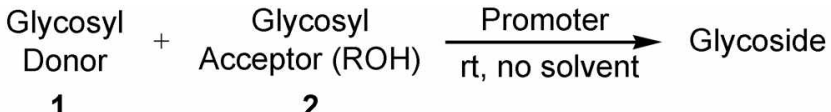

1

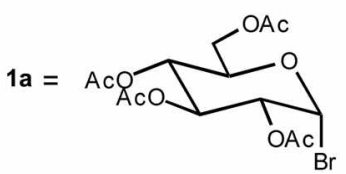

2a, $\quad \mathrm{R}=\mathrm{CH}_{3}$

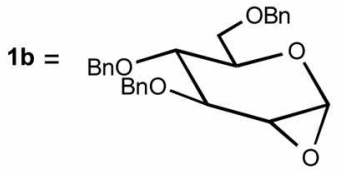

2b, $\quad \mathrm{R}=$
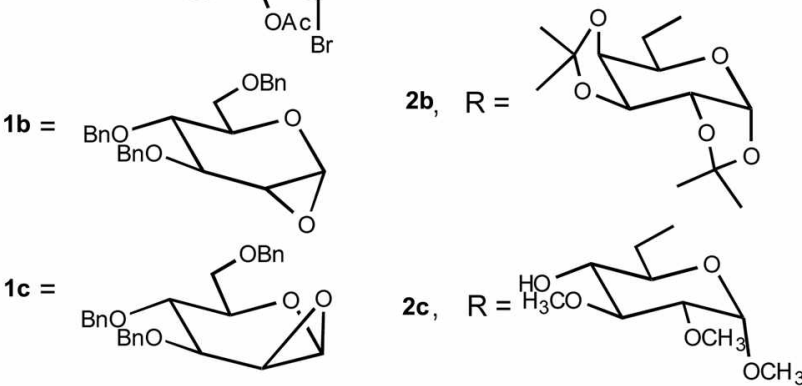

Scheme 1. $O$-Glycosylation of various acceptors with donors in the solid to solid state.

producing $32 \%$ and $18 \%$ of product, respectively. When liquid acceptor $2 \mathbf{a}$ and a solution of $2 \mathbf{b}$ in $\mathrm{CHCl}_{3}$ were added to the ground mixture of $\mathbf{1 b}$ and an activator, glycosylation also proceeded. These observations clearly demonstrate that solid to solid state methodology could be widely extended in glycosylation.

In summary, although there is still room for improvement in yield of the product, the solid to solid state reaction methodology could effectively serve as a simple, mild, and stereoselective procedure in glycosylation.

\section{Experimental Section}

General procedure for glycosylation in the solid to solid state 1b-2c: To a mixture of 1,2-anhydro-3,4,6-tri- $O$ benzyl- $\alpha$-D-glucopyranose (1b, $107 \mathrm{mg}, 0.247 \mathrm{mmol}$ ), methyl 2,3-di- $O$-methyl- $\alpha$-D-glucopyranose $(2 \mathrm{c}, 55 \mathrm{mg}$, $0.25 \mathrm{mmol}$ ), and $\mathrm{ZnCl}_{2}$ ( $3 \mathrm{mg}, 0.022 \mathrm{mmol}$ ) was ground with a mortar and pestle at room temperature for $30 \mathrm{~min}$ under argon atmosphere in a glove box. After quenching the reaction by the addition of water, $\mathrm{CHCl}_{3}$ was added. The reaction mixture was filtered and extracted with $\mathrm{CHCl}_{3}$. Then combined extracts were concentrated in vacuo and the residue was chromatographed (Silica gel 60, Ethyl acetate: $\mathrm{n}$-Hexane/3:1) to produce $70.1 \mathrm{mg}$ of disaccharide $(43 \%)$ as well as $60.0 \mathrm{mg}$ of $3,4,6$-tri- $O$-benzyl- $\alpha / \beta$-D-glucose $(55 \%)$. 
Table 1. $O$-Glycosylation in the solid to solid state

\begin{tabular}{|c|c|c|c|c|c|c|}
\hline Substrate & Reactant & Method & Activator & Product & $\begin{array}{c}\text { Yield } \\
(\%)\end{array}$ & $\begin{array}{l}\text { Ratio } \\
\text { (a:b) }\end{array}$ \\
\hline \multirow[t]{4}{*}{$1 \mathrm{a}$} & $2 b$ & solid & A & $1 a-2 b^{5}$ & 29 & $\beta$ \\
\hline & $2 b$ & solution & A & $1 a-2 b$ & 19 & $\beta$ \\
\hline & $2 c$ & solid & A & $1 a-2 c$ & 21 & $\beta$ \\
\hline & $2 c$ & solution & A & $1 a-2 c$ & 65 & $\beta$ \\
\hline \multirow[t]{7}{*}{$1 b$} & $2 a$ & solid" & B & $1 b-2 a^{6}$ & 99 & $\beta$ \\
\hline & $2 b$ & solid ${ }^{\prime}$ & B & $1 b-2 b^{7}$ & 37 & $\beta$ \\
\hline & $2 b$ & solutiont & B & $1 b-2 b$ & 21 & $\beta$ \\
\hline & $2 c$ & solid & B & $1 b-2 c$ & 43 & $\beta$ \\
\hline & $2 c$ & solutiont & B & $1 b-2 c$ & 35 & $\beta$ \\
\hline & $2 c$ & solid & $\mathrm{C}$ & $1 b-2 c$ & 32 & $\beta$ \\
\hline & $2 c$ & solid & $\mathrm{D}$ & $1 b-2 c$ & 18 & $\beta$ \\
\hline \multirow[t]{4}{*}{ lc } & $2 b$ & solid & B & $1 c-2 b^{7}$ & 33 & $\alpha$ \\
\hline & $2 b$ & solution $^{g}$ & B & $1 c-2 b$ & 26 & $\alpha$ \\
\hline & $2 \mathfrak{c}$ & solid & B & $1 c-2 c$ & 35 & $\alpha$ \\
\hline & $2 c$ & solutions & B & $1 c-2 c$ & 39 & $\alpha$ \\
\hline
\end{tabular}

"A, AgOCOCF $; 3, \mathrm{ZnCl}_{2} ; \mathrm{C}_{1}$ Amberlite $\mathrm{IRC}-50$, II form; D, no aclivator but MS4A. ${ }^{b}$ All products gave satisfactory ${ }^{\prime} \mathrm{H}-$ and ${ }^{13} \mathrm{C}-\mathrm{N} M \mathrm{R}$ specira. 'Determined after isolation. "The reaction was perfomed in $\mathrm{CII}, \mathrm{Cl}$ at $-78^{\circ} \mathrm{C}$ for $3 \mathrm{hr}$, then stirred at rt for $3 \mathrm{hr}$. "After substrate and activator were ground with a mortar and pestle for $20 \mathrm{~min}$, one drop of liquid acceptor was added then the mixture was further ground for an additional $20 \mathrm{~min}$. Afler substrate $\mathbf{1 b}$ and activalor were ground in an agate mortar for $20 \mathrm{~min}$, one drop of solution containing $\mathbf{2 b}$ in $\mathrm{Cl} \mathrm{ICl}_{3}$ was added, and the resulting mixture was ground for an additional 20 min. ${ }^{2}$ Reaction was performed in THF at $-78^{\circ} \mathrm{C}$ for $2 \mathrm{hr}$, then slitred at tt for $24 \mathrm{hr}^{\text {? }}$

General procedure for glycosylation in solution state 1c-2e: A solution of 1,2-anhydro-3,4,6-tri- $O$-benzyl- $\beta$-Dmannopyranose (1c, $38.5 \mathrm{mg}, 0.089 \mathrm{mmol}$ ) and methyl $2,3-$ di- $O$-methyl- $\alpha$-D-glucopyranose (2c, $31.7 \mathrm{mg}, 0.143 \mathrm{mmol}$ ) in THF $(0.30 \mathrm{~mL})$ was stirred at $-78^{\circ} \mathrm{C}$, then $0.2 \mathrm{M} \mathrm{ZnCl}_{2}$ in diethyl ether $(0.75 \mathrm{~mL}, 0.022 \mathrm{mmol})$ was added and stirred at $-78^{\circ} \mathrm{C}$ for $2 \mathrm{hr}$ and allowed to warm over $1 \mathrm{hr}$ to $\mathrm{rt}$. After stirring at rt for $24 \mathrm{hr}$. The reaction mixture was quenched by the addition of water, $\mathrm{CHCl}_{3}$ was added. The reaction mixture was filtered and extracted with $\mathrm{CHCl}_{3}$. Then combined extracts were concentrated in vacuo and the residue was chromatographed (Silica gel 60 , Ethyl acetate: $n$-Hexane 13:1) to produce $22.73 \mathrm{mg}$ of disaccharide (39\%).

Disaccharide 1a-2c (Table 1): $\mathrm{mp}: 53-55^{\circ} \mathrm{C}$; $[\alpha]_{\mathrm{D}}^{20}$ : $45.6^{\circ}\left(\mathrm{c}=5.0\right.$ in $\left.\mathrm{CHCl}_{3}\right) ;{ }^{1} \mathrm{H}-\mathrm{NMR}\left(\mathrm{CDCl}_{3}\right): \delta 2.0(\mathrm{~m}, 12 \mathrm{H})$, $2.55(\mathrm{~b}, \mathrm{OH}), 3.3-3.6(\mathrm{~m}, 9 \mathrm{H}), 4.6\left(\mathrm{~d}, J=7.7 \mathrm{~Hz}, 1 \mathrm{H}, \mathrm{C}_{1}-\mathrm{H}\right)$, $4.8\left(\mathrm{~d}, 1 \mathrm{H}, \mathrm{C}^{\prime}{ }_{1}-\mathrm{H}\right) ;{ }^{13} \mathrm{C}-\mathrm{NMR}\left(\mathrm{CDCl}_{3}\right): \delta 170.8,170.3$,
$169.4,169.3,101.0,97.34,82.6,81.7,72.7,71.8,71.1,70.6$ $69.9,68.6,68.3,61.8,61.2,58.5,55.2,20.75,20.70,20.62$; Anal. Caled for $\mathrm{C}_{23} \mathrm{H}_{36} \mathrm{O}_{15}: \mathrm{C}, 50.00 ; \mathrm{H}, 7.03$. Found: $\mathrm{C}$, $49.96 ; \mathrm{H}, 6.92$.

Disaccharide 1b-2c (Table 1): Syrup; $[\alpha]_{D}^{20}: 47.8^{\circ}(\mathrm{c}=$ 3.20 in $\mathrm{CHCl}_{3}$ ); ${ }^{1} \mathrm{H}-\mathrm{NMR}\left(\mathrm{CDCl}_{3}\right): \delta 2.7(\mathrm{br}, 2 \mathrm{H}), 3.25$ (dd, $1 \mathrm{H}), 3.43(\mathrm{~s}, 3 \mathrm{H}), 3.49(\mathrm{~s}, 3 \mathrm{H}), 3.64(\mathrm{~s}, 3 \mathrm{H}), 3.33-3.90(\mathrm{~m}$, $9 \mathrm{H}), 4.17$ (dd, $1 \mathrm{H}), 4.36(\mathrm{~m}, 1 \mathrm{H}), 4.40-4.60(\mathrm{~m}, 3 \mathrm{H}), 4.67$ (d, $\left.J=8.37 \mathrm{~Hz}, 1 \mathrm{H}, \mathrm{C}_{1}-\mathrm{H}\right), 4.78-4.97(\mathrm{~m}, 4 \mathrm{H}), 7.13-7.35(\mathrm{~m}$, $15 \mathrm{H}$, aromatic); ${ }^{13} \mathrm{C}-\mathrm{NMR}\left(\mathrm{CDCl}_{3}\right): \delta 138.5,138.0,137.9$, $128.5,128.4,128.3,128.0,127.9,127.8,127.7,127.6$, $103.4,97.5,84.5,82.7,81.7,77.2,75.19,75.0,74.4,73.5$, $70.5,69.9,69.2,68.8,61.3,58.6,55.3$; Anal. Calcd for $\mathrm{C}_{33} \mathrm{H}_{46} \mathrm{O}_{11}: \mathrm{C}, 66.04 ; \mathrm{H}, 7.08$. Found: $\mathrm{C}, 65.96 ; \mathrm{H}, 6.98$.

Disaccharide 1c-2c (Table 1): Syrup; $[\alpha]_{\mathrm{D}}^{20}: 50.9^{\circ}(\mathrm{c}=$ 1.35 in $\mathrm{CHCl}_{3}$ ); ${ }^{1} \mathrm{H}-\mathrm{NMR}\left(\mathrm{CDCl}_{3}\right): \delta 2.3(\mathrm{br}, 2 \mathrm{H}$ ), 3.23 (dd, $1 \mathrm{H}), 3.40(\mathrm{~s}, 3 \mathrm{H}), 3.51(\mathrm{~s}, 3 \mathrm{H}), 3.63(\mathrm{~s}, 3 \mathrm{H}), 3.41-3.9(\mathrm{~m}$, $8 \mathrm{H}), 4.08$ (dd, 1H), 4.14 (1H), 4.42 (t, 1H), 4.44-4.83 (m, $6 \mathrm{H}, \mathrm{BnCH}_{2}$ ), 4.86 (d, $\left.J=3.5 \mathrm{~Hz}, 1 \mathrm{H}, \mathrm{C}^{\prime}{ }_{1}-\mathrm{H}\right), 4.95$ (d, $J=$ $\left.1.38 \mathrm{~Hz}, 1 \mathrm{H}, \mathrm{C}_{1}-\mathrm{H}\right), 7.10-7.35\left(\mathrm{~m}, 15 \mathrm{H}\right.$, aromatic); ${ }^{13} \mathrm{C}-$ NMR $\left(\mathrm{CDCl}_{3}\right): \delta 137.9,137.7,128.5,128.4,128.3,128.0$, $127.9,127.8,127.7,99.3,97.5,82.9,81.6,80.2,75.2,74.3$, $73.3,72.0,71.3,70.1,69.4,68.7,68.3,56.7,61.3,58.7,55.2$; Anal. Calcd for $\mathrm{C}_{36} \mathrm{H}_{46} \mathrm{O}_{11}: \mathrm{C}, 66.04 ; \mathrm{H}, 7.08$. Found: $\mathrm{C}$, $65.96 ; \mathrm{H}, 7.12$.

\section{References}

I. (a) Schmidi, R. R. Pure Appl, Chem. 1989, 61, 1257, (b) Paulsen, H. Angew, Chem, Int. Ed. Engl. 1990, 29, 823. (c) Toshima, K,; Tatauda, K. Chem. Rev. 1993, 93, 1503. (d) Danishefsky, S. J.; Bilodeau, M. T. Angew: Chem. Int. Ed. Engl. 1996, 35, 1380.

2. Im. J.; Kim, J.; Kim. S.; Hahn, B.; Toda, F. Tetrahedron Lett. $1997,38,451$

3. (a) Eby, R.; Srivasta, V. Carbohydr: Res, 1982, 102, I. (b) Halcomb, R. L.; Danishefsky, S. J. J. Ani. Chent. Soce 1989, MI, 6661.

4. Sondheimer, S.; Yamaguchi, H.; Schuerch, C. Carbohydr. Res. 1979. 74.327.

5. (a) Kochetkov, N. K.; Khorlin, A. J.; Bochkov, A. F. Tetrahedron 1967, 23, 693. (b) Hanessian, S.; Banoub, J. Carbolydi: Res. $1977,53, \mathrm{C}-13$.

6. (a) Crich, D.; Lim, L. B. L. J. Chem. Soc., Perkin Trans. $/ 1991$, 2209.; (b) Ekborg. G; Lindberg, B.; Lonngren, J. Acta Chem. Scand. 1972, 26, 3287 .

7. (a) Nicota, F.; Panza, L.; Rochetti, F.; Russo, G; Toma, L. $J$. Chem. Soc., Perkin Trans, 1 1987, 1319. (b) Boren, H. B.; Ekborg, G.; Eklind, K.; Garegg, P. J.; Pilotti, A.; Shwahn, C. G Acta Chem. Scand, 1973, 27, 2639 . 